\title{
Editor's Note: Vascular Medicine and Surgery (Vol 5 Iss 4)
}

\section{Sy Duong-Quy ${ }^{1,2 *}$}

${ }^{1}$ Department of Cardio-Pulmonology Functional Testing, Cochin Hospital, Paris Descartes University, France ${ }^{2}$ Penn State College of Medicine, USA

Vascular medicine deals with any/all discrepancies related to the structural and functional aspects of vessels (veins and arteries). Owing to the multifaceted physiological association of blood and lymph vessels, the discrepancies caused due to their improper functioning are also diverse. Some of the most common examples correspond to pathological complications of dysfunction of blood circulation system as well as immunological implications of improper circulation of non-hemoglobin blood components. The involved treatment strategy can be ranged from administration of pharmaceutical therapies in combination with invasive surgeries.

The Journal of Vascular Medicine \& Surgery works for the dissemination of research advancements that pertains to the above discussed aspects. The current issue publishes many such articles and a brief overview of which is summarized in this Editor's notes.

Standardization of treatment methods for attaining best possible clinical outcomes is a mandatory aspect of medical sciences. This concept is further realized when a specific condition is known to be treated with available standardized drugs as well as contemporary interventional techniques. In such conditions, it has been observed that the medical community has different opinions regarding the optimal strategy of treatment. One such condition is the treatment of chronic obstructive pulmonary disease (COPD). The article published by TranVan et al. thus presented a detailed study that made a comparative assessment of the application of long-term oxygen therapy (LTOT) vs. LTOT and vasodilator drugs viz. sildenafil (PDE-5 inhibitor) and simvastatin (HMG CoA reductase inhibitor) [1]. The study most specifically studied the effect of the both on the possible changes in the pulmonary arterial pressure of COPD patients with hypoxia. The systolic pulmonary arterial pressures (PAP) and diffusing capacity of the lungs for carbon monoxide (DLCO) were significantly ameliorated after 3 to 6 months. The results obtained by this study suggest that mean systolic PAP of COPD patients administered with LTOT plus sildenafil and LTOT combined with sildenafil and simvastatin were significantly lower (41 $\pm 9 \mathrm{mmHg}$ and $39 \pm 7 \mathrm{mmHg}$, respectively) than that of those administered with LTOT alone $(46 \pm 10 \mathrm{mmHg})$. The follow-up studies further validated that application of LTOT in conjunction with sildenafil and simvastatin have additional therapeutic benefits. Hence, it was concluded that their application should be promoted on large scale clinical settings.

The article published by Gamal and Mohamed conducted a study that was intended to test the clinical efficacy of ultrasound guided foam sclerotherapy (UGFS), a contemporary method of treatment of varicose veins. The study was aimed to make a comparative assessment of therapeutic efficacy of the conventionally applied surgical treatment method with that of UGFS. It was observed that post treatment parameters viz. total occlusion of great saphenous vein (GSV), recurrence rate and patient satisfaction were almost similar in both the groups. The author used the data to validate that UGFS is as efficient as the surgical treatment method and can be used to replace the conventional surgical interventions if and when required [2].

The article published by Belczak et al. was based on management of uterine myomas, especially the symptomatic improvement of the disease. The authors made an effort to validate the therapeutic efficacy of less invasive treatment methods of uterine artery embolization (UAE) [3]. In order to do so, the authors studied the symptomatic changes in thirty-one women who underwent UAE. Scaling the intensity of the discomforting symptoms of abdominal pain, swelling and bleeding during and out of menstrual periods as well as experiencing pain during sexual intercourse pre- and post-UAE indicated that bleeding out of menstrual periods score was significantly increased after UAE while the score for most other symptoms decreased. It was thus concluded that UAE provides extremely positive outcomes, especially in women who are characterized with higher abdominal pain and bleeding scores.

Yet another article that presented a study aimed towards evaluating the therapeutic efficacy of an interventional method was published in the current issue of the article. The article published by Vo-Thi-Kim, et al. described the correlations between the occurrences of obstructive sleep apnea in hypertensive patients with refractory high blood pressure (R-HBP) [4]. Furthermore, the authors also made an effort to check for the therapeutic efficacy of administration of continuous positive airway pressure (CPAP) in managing high blood pressure. This original research encompassed study of outcome of RHBP patients with OSA (apnea-hypopnea index (AHI) $>30 /$ hour) after they were treated with CPAP. It was observed that there occurred significant reduction in the systolic-BP and diastolic-BP as observed up to after 3 months of being administered with CPAP. Integrative analysis of the changes observed in the Epworth score, fasting glucose, total and LDL cholesterol also indicated positive results after the patients were administered with CPAP. It was therefore recommended that this method can be applied for the treatment of severe OSA in R-HBP patients.

The article published by Ando et al. presented a case report of an anuloaortic ectasia patient who underwent localized dissection in the right Valsalva sinus [5]. The clinical specifications of the patient indicated that he initially underwent aortic root replacement (mechanical valve) and was later re-admitted for mediastinitis. Furthermore, though $\mathrm{C}$ reactive protein (CRP) levels of blood serum were found to be normal, it was observed that his body temperature rose to $40^{\circ} \mathrm{C}$, after which he underwent emergency re-sternotomy. During the surgery he went into shock as a proximal anastomotic site got ruptured. Management of the condition was done by doing a cardiopulmonary bypass and second aortic root replacement. Nine days after, his chest wound started bleeding, due to which he was again

*Corresponding author: Sy Duong-Quy, Professor, MD, PhD, FCCP, Cochin Hospital, Paris Descartes University, Penn State Medical College, USA, Tel: +33679193377; E-mail: sduongquy.jfvp@gmail.com

Received December 11, 2017; Accepted December 15, 2017; Published December 20, 2017

Citation: Duong-Quy S (2017) Editor's Note: Vascular Medicine and Surgery (Vol 5 Iss 4). J Vasc Med Surg 5: e121. doi: 10.4172/2329-6925.1000e121

Copyright: ( 2017 Duong-Quy S. This is an open-access article distributed under the terms of the Creative Commons Attribution License, which permits unrestricted use, distribution, and reproduction in any medium, provided the original author and source are credited. 
administered with a cardiopulmonary bypass. Laceration was found at the same annular spot and interrupted sutures were inserted in the left cardiac muscle. The next step involved implantation of Freestyle aortic root bioprosthesis and anastomosis of another prosthetic vessel. The complexity of the clinical procedure described in the article is the main factor that makes it a crucial reference material for physicians who may be faced with similar clinical cases in future.

Another case report that was authored by Nonga et al. was based on a clinical case of pulmonary embolism in a 30 years old male patient [6]. While the condition has already been established to have massive medical burden, the only positive silver lining is that it is low treatable via the standardized surgical treatment method that has gradually evolved as the safest and hence the most commonly applied therapeutic option. The case reported in here was also treated with the help of thrombectomy and lung resection. It also marks the first ever case of pulmonary embolism treatment in Cameroon to have successfully accomplished the procedure.

On the other hand, the case report published by Lee et al. presented a rare case of peripherally inserted central catheter (PICC) [7]. The rarity of the case lies in the fact that the post-procedure chest X-ray located the catheter to be present in the pericardiacophrenic vein of the 82 -year-old male patient. The patient was being treated for severe sepsis with shock, during which PICC was placed as a method of providing vasopressor support. The clinically risky positioning of the catheter acts as a reminder of the complexity of the medical procedures. The information presented in the article may further be implicated for educating young physicians regarding the possible complications of the procedure and how to avoid them.
The current issue fulfilled its purpose of enriching its readers with avant garde research manuscripts that are based on scientific achievements as well as clinical aspects of vascular medicine. The journal remains committed to its purpose of dissemination of high quality scientific manuscripts from the field of vascular medicine.

\section{References}

1. Tran-Van H, Vo-Thi-Kim A, Tran-Ngoc T, Duong-Quy S (2017) Comparison of Long Term Oxygen-Therapy (LTOT) and LTOT Combined with Sildenafil and Simvastatin in the Treatment of Severe Chronic Obstructive Pulmonary (COPD) with Hypoxia at Rest and Severe Pulmonary Arterial Hypertension. J Vasc Med Surg 5: 324

2. Gamal WM, Mohamed AF (2017) Duplex Guided Foam Sclerotherapy Versus Surgery in Management of Primary Varicose Veins. J Vasc Med Surg 5: 325

3. Belczak SQ, Szejnfeld D, de Almeida Cardoso N, Klajner RK, Ogawa LC, et al. (2017) Symptoms Improvement after Uterine Artery Embolization for Myomas Management. J Vasc Med Surg 5: 326.

4. Vo-Thi-Kim A, Nguyen-Xuan B, Dao-Van D, Duong-Quy S (2017) The Effect of Continuous Positive Airway Pressure (CPAP) in Treatment of Patients with Refractory High Blood Pressure Associated with Severe Obstructive Sleep Apnea (OSA). J Vasc Med Surg 5: 327.

5. Ando T, Akiyama D, Okada H, Takeda M (2017) Successful Management of Refractory Prosthetic Vessel Infection, Prosthetic Valve Endocarditis and Mediastinitis: Report of a Case-Late Timing Caused Repeated Surgery. J Vasc Med Surg 5: 328

6. Nonga BN, Ze JJ, Messomo D, Handy DE, Pondy AO, et al. (2017) Thrombectomy and Lung Resection for Massive Left Pulmonary Embolism in a 30 Years Old Man in Yaoundé, Cameroon. J Vasc Med Surg 5: 329

7. Lee YI, Miyakawa L, Bergman M, Smith RL (2017) PICC'ing Your Way into the Pericardium: A Potential Catastrophic Complication of a PICC Line. J Vasc Med Surg 5: 330. 